\title{
INTENSITIES OF WEAK LINES AT THE SOLAR LIMB
}

\author{
J. Houtgast and D. Koelbloed \\ Sterrewacht Sonnenborgh, Utrecht, and Sterrenkundig Instuut, Amsterdam, Holland
}

\section{OBSER VATIONS}

From spectra in the regions $4588-4682 \AA$ and $4783-4873 \AA$ taken during the eclipse of 1961 February 15 at Brac, Yugoslavia(1) total intensities of weak lines were determined in the Fraunhofer spectrum of the Sun's limb and in the emission spectrum of the low chromosphere. It appears that:

(1) the decrease of the equivalent widths of all lines towards the limb goes slowly and in practically the same way for all lines and that only for $r_{\odot}>0.995$ a faster decrease sets in;

(2) lines of different origin behave differently in the transition photosphere-chromosphere;

(3) of the lines, thus far investigated, atomic lines are weak and ion lines are relatively strong in emission in the low chromosphere.

From the observations, made with a slitless spectrograph and which were standardized by means of the light from the centre of the Sun's disk, the line strengths were determined in absolute units. For the absorption lines this strength means the amount of radiation missing from the continuous spectrum because of the presence of the absorption line. For emission lines it has the usual meaning. All absolute intensities given here are integrated ones, as observed with the slitless spectrograph and refer to the radiation from a slice of $1 \mathrm{~cm}$ width, through photosphere and chromosphere, parallel to the dispersion in the spectrum, extending outwards from the level where the Moon's limb is projected, expressed in $10^{14}$ ergs per sec per sterad per Ångstrom.

In Fig. 1 the intensity is plotted for the line Fe 4611.295 $\AA$ and in this case the equivalent width is given too. The value of the equivalent width in the centre of the Sun is indicated at $131 \mathrm{~m} \AA$, which value is taken from the Utrecht Catalogue. ${ }^{(2)}$ This line is by far the strongest line treated in this communication, but by means of the curves in Fig. 1 , the absolute intensities of the lines in the next figures can be transformed to equivalent widths. This is so because the absolute intensity in the solar spectrum in the wavelength region covered, is constant within 1 per cent. ${ }^{(3)}$

In all our figures the solar limb is placed as well as possible, in the position determined by DE GROOT ${ }^{(4)}$ from photoelectric measurements during the same eclipse.

The relation between the scales of $r_{g}$, the number of $\mathrm{km}$ within the Sun's limb and the time in which the Moon's edge moved over a certain distance, is indicated in Fig. 7 along the abscissa.

In Fig. 2 absolute intensities of a number of Fe lines are given. The decrease of the 


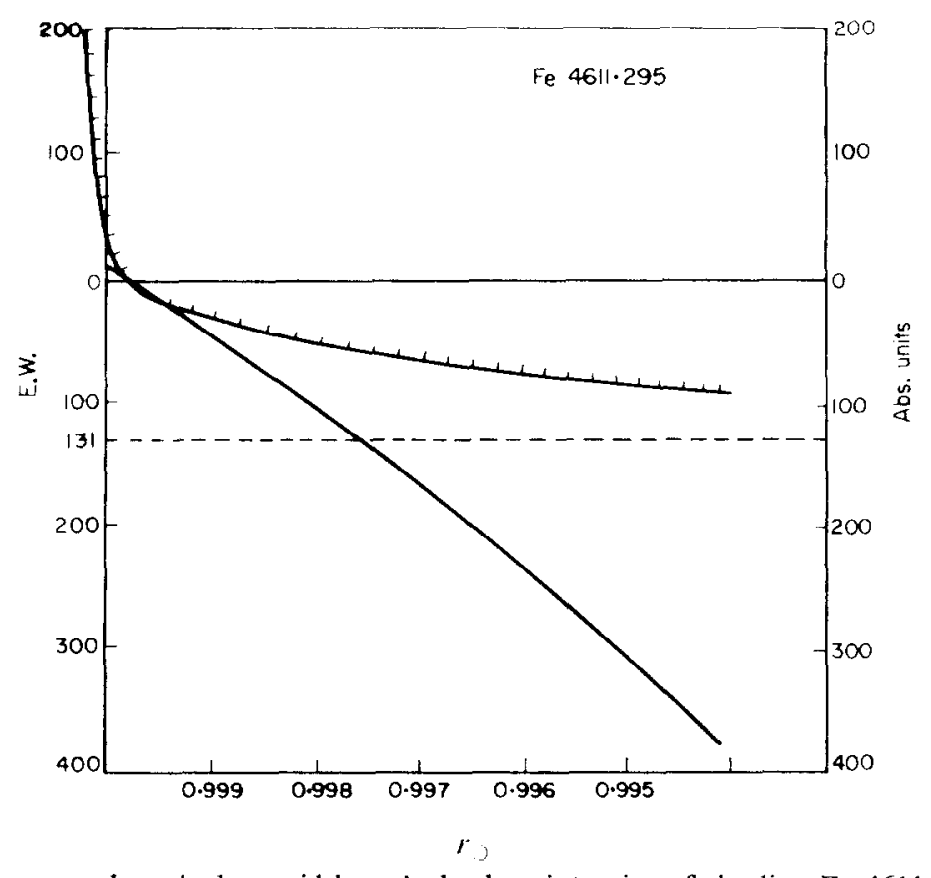

FIG. 1. Integrated equivalent width and absolute intensity of the line Fe $4611.295 \AA$ as derived from the eclipse observations. The value of the equivalent width for the centre of the Sun is indicated at $131 \mathrm{~m} \AA$.

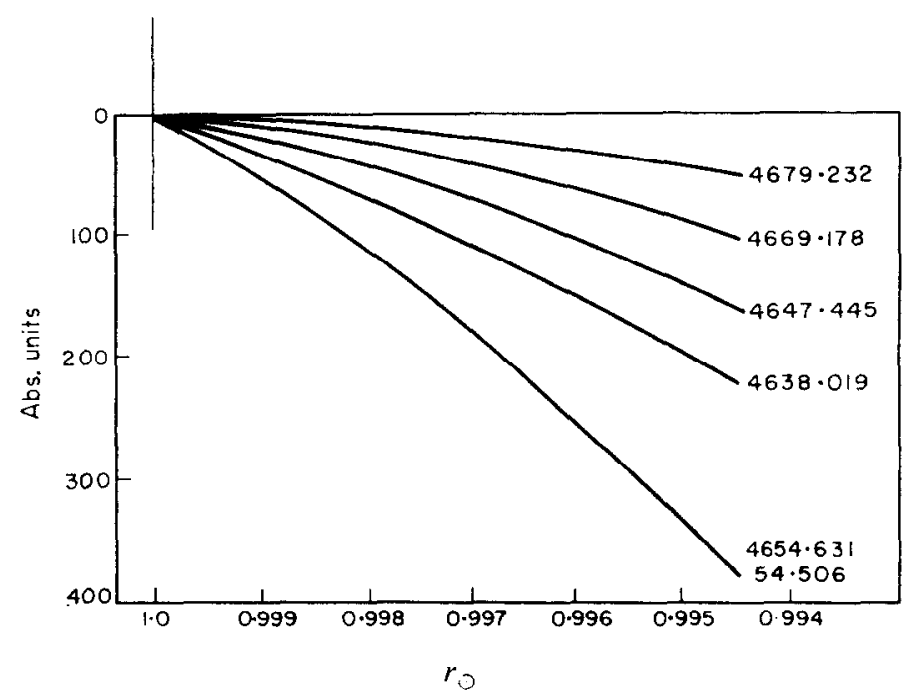

FIG. 2. Integrated absolute intensities of $5 \mathrm{Fe}$ lines of different strengths as a function of $r$ 
intensities towards the limb is, relatively, nearly the same; all $\mathrm{Fe}$ lines so far investigated, show this behaviour in the same manner.

Figure 3 shows the curve for the line $\mathrm{Na} 4668 \cdot 576 \AA$, together with the measured points, to give an impression of the scattering. The exposure times vary from $0^{s} \cdot 1^{\text {to }} 1^{s}$, and for one of the points to $3^{s}$. To get a higher resolution in time would be very desirable at next eclipses. The points near $r_{\odot}=1$ had to be determined from different strips in the spectrum, i.e. from different points of the chromosphere.

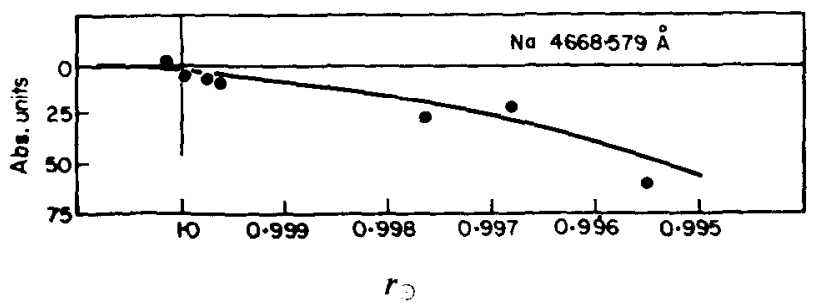

Fig. 3. Integrated absolute intensity of the line $\mathrm{Na} 4668 \cdot 576 \AA$.

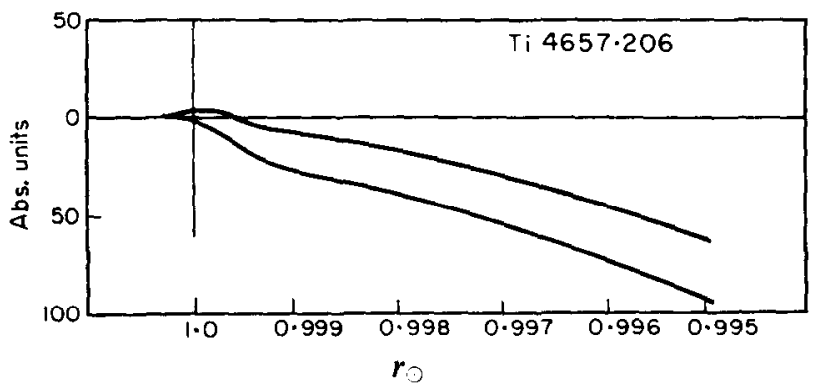

FIG. 4. Integrated absolute intensity of the line $\mathrm{Ti}^{+} 4657 \cdot 206 \AA$ as observed and this curve diminished by the emission of the chromosphere according to one of the curves of Fig. 7.

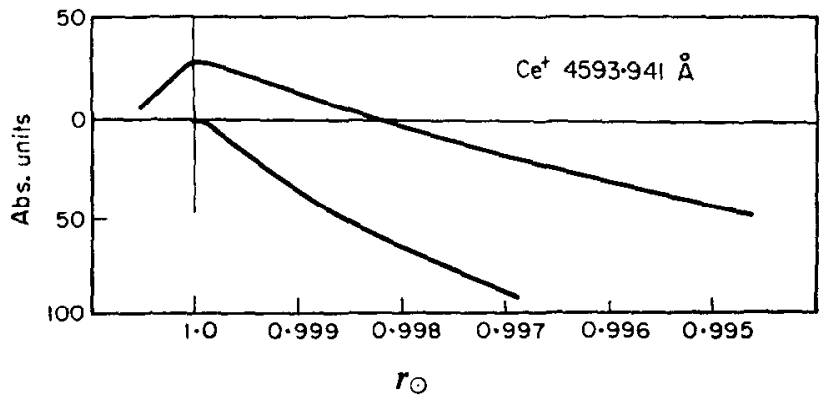

FIG. 5. The same as in Fig. 4 but for the line $\mathrm{Ce}^{+} 4593.941 \AA$.

The behaviour of some ion lines is shown in Figs. 4, 5 and 6. We draw attention to the difference between both groups of lines (of atoms and of ions), just inside and just outside the Sun's limb. Generally atomic lines change from absorption to emission at the very limb, whereas the ion lines already within the limb show an emission maximum. 


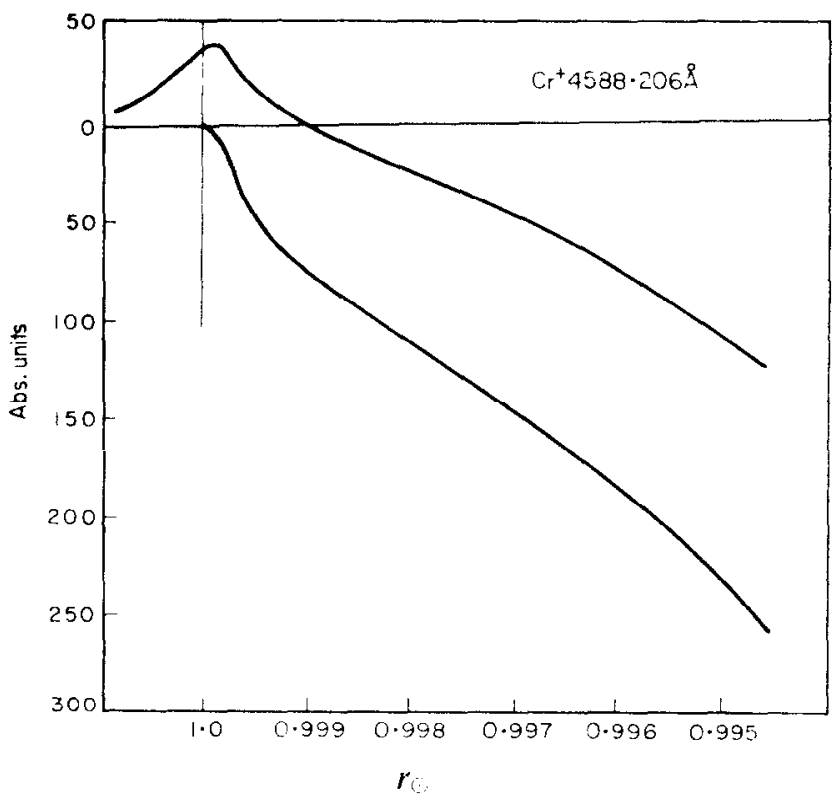

Fig. 6. The same as in Fig. 4 but for the line $\mathrm{Cr}^{+} 4588 \cdot 206 \AA$.

This difference is contrary to the nearly equal behaviour as absorption lines in the spectrum of the photosphere within $0.995 r_{0}$ and also to the intensity decrease in the higher chromosphere. As to the latter point, Houtgast ${ }^{(5)}$ found practically no difference between gradients of lines of Fe I, Ti I and Ti II in the region of the chromosphere between the heights of 200 and $2500 \mathrm{~km}$.

\section{SPECTRAL LINES FORMED IN THE PHOTOSPHERE}

To obtain the intensities of lines as they are formed in the photosphere, the chromospheric emission was subtracted. The emission per $\mathrm{cm}^{3}$ as a function of height in the chromosphere was, because of lack of more accurate data, set equal to:

$$
i(h)=i(0) \cdot \exp (-\alpha h),
$$

in which for $\alpha$ the value $4 \cdot 10^{-8} \mathrm{~cm}^{-1}$ was substituted. If $h$ is expressed in units of $1000 \mathrm{~km}$, then $\alpha=4$. The integrated intensity up to a certain value of $r_{\circ}$, as computed with the formula, is drawn in Fig. 7 for different intensities. For each of the observed lines, for which this was possible, the theoretical curve was chosen for which the form outside the limb coincides with the observational curve. It is clear that this only could be done for the ion lines.

After subtraction of the chromospheric emission the photospheric absorption remains, which as such has to be explained by a photospheric model with selective and continuous absorption. The curves, representing this, have been drawn in Figs. 4-6.

The difficulty has always been to explain why absorption lines have their observed strengths near the limb (REDMAN ${ }^{(6)}$; UNSÖLD $\left.{ }^{(7)}\right)$. Since, after correction for chromospheric 


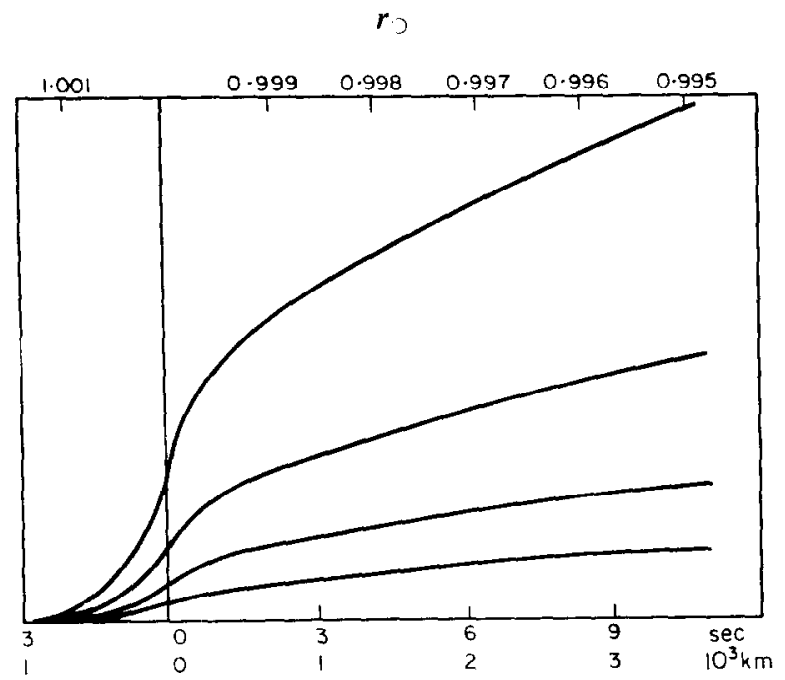

FIG. 7. Computed integrated emission of the chromosphere according to the formula $i(h)=i(0) \exp (-\alpha h)$.

emission the absorption lines are stronger than is observed directly, the discrepancy between observation and theory appears to have still increased.

We should like to compute, based on a simple model, the values of equivalent width and absolute integrated intensity of a hypothetical weak spectral line, as a function of the distance from the Sun's limb in the region outside $0.995 r_{C}$.

Instead, however, of a line profile and its equivalent width, we consider the intensity $i$, relative to the continuum caused by selective absorption, adopting a ratio between selective and continuous absorption of 0.05 . This ratio is assumed to be constant throughout the photosphere. The computation is carried out with black-body radiation $E=E_{0}(1+\beta \tau), \beta$ taken equal to 3 .

Curvature of the photospheric layers was taken into account; the solar $\operatorname{limb}\left(r_{\odot}=1\right)$ was supposed to coincide with tangential optical depth $\tau=1$; the model was adapted to the limb-intensity gradient, obtained by DE GROоT ${ }^{(4)}$ and the absorption coefficient was assumed to increase linearly with depth.

In the region near the limb we obtain the following results for $i$, the absorption $(-)$ or emission $(+)$ relative to the continuum:

$\begin{array}{llcccr}r_{\supset}: & 1.00029 & 1.00014 & 0.99985 & 0.99957 & 0.99914 \\ i: & +0.032 & +0.022 & +0.004 & -0.002 & -0.004\end{array}$

The Milne-Eddington model, without taking into account the curvature of the photospheric layers and with a sharp limb at $\bar{r}_{\supset}=1$, gives at

$\begin{array}{lccc}r_{\odot}: & 0.99914 & 0.995 & 0\left(\text { centre }_{\odot}\right) \\ i: & -0.005 & -0.011 & -0.039\end{array}$


These values connect continuously to those of the foregoing table: for $r<0.999$ the curvature and the lack of sharpness of the limb may be neglected.

Further we computed the integrated equivalent widths in several points beyond $r=0.995$; the connecting curve can be compared with the observations in Fig. 1 . Relative to the line strength in the centre of the Sun, the computed values of the absorption are much smaller than observed.

Finally we computed the integrated absolute intensities, the curve also being drawn in Fig. 8 .

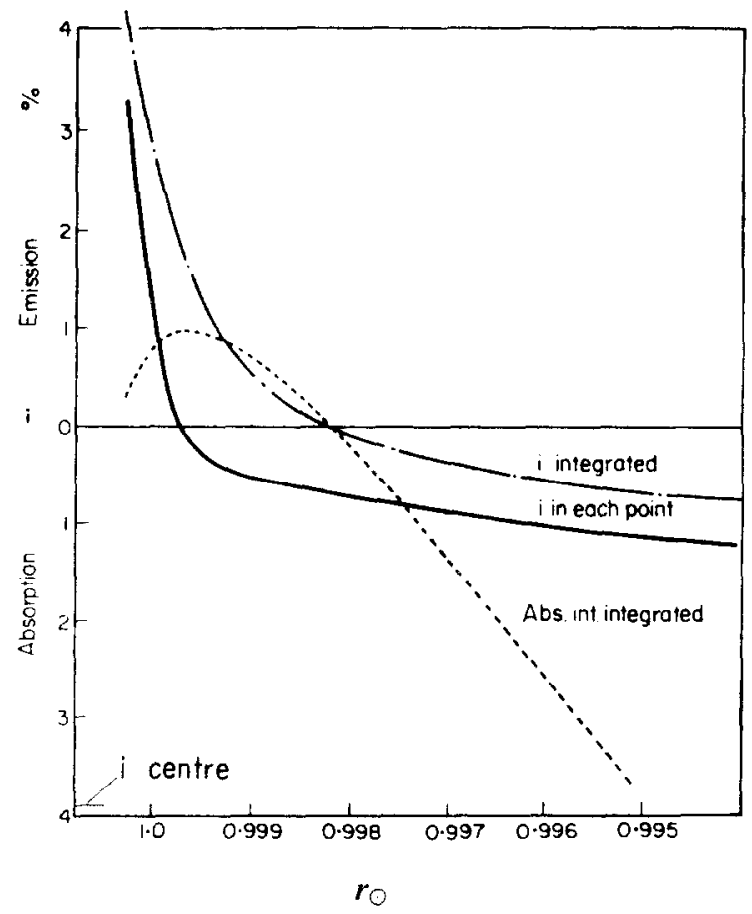

FIG. 8. Equivalent width and integrated absolute intensity according to the photospheric model as described in the text.

Our model results in integrated values which change from absorption into emission at about $r=0.9982$. The curve of integrated absolute intensities in Fig. 8 has some resemblance with the curves in Figs. 4-6, especially that in Fig. $5\left(\mathrm{Ce}^{+4593.941} \AA\right)$ but we have to remind ourselves that the latter curves include the emission of the chromosphere, which in Fig. 8 was not taken into account.

Acknowledgement-The funds for the eclipse expedition and for the visit of one of the authors to this colloquium were granted by the Netherlands Organization for Pure Research (Z.W.O.).

For their help in this investigation we thank Miss A. I. Nekkers (photometry) and Messrs. A. J. van Vloten (working out of general computations) and $J$. Visch (computations and figures). 


\section{REFERENCES}

1. J. Houtgast, Proc. Acad. Sci. Amst. B 65, 313 (1962).

2. Preliminary Photometric Catalogue of Fraunhofer Lines $3164 \AA-8770 \AA$. Rech. astr. Obs. Utrecht (1960).

3. D. LABS. and H. NeCkEL, Astrophys. J. 135, 969 (1962); and Z. Astrophys. 55, 269 (1962).

4. T. DE Groot, Bull. Astr. Inst. Ned. 16, 181 (1962).

5. J. Houtgast, Rech. ast . Obs. Utrecht 13, 32 (1957).

6. R. O. Redman, Mon. Nat. R, Astr. Soc. 103, 173 (1943).

7. A. Uvsöld, Physik der Sternatmosphären, p. 448 (1955).

\section{DISCUSSION}

R. N. Thomas: I do not agree with the idea of subtracting out the "chromospheric contribution". One should treat the atmosphere as a whole in discussing the line formation. The value of $I_{v}$ at some point in the atmosphere--say at a point called the top of the photosphere-will differ depending whether there is further atmosphere lying above that point or not. So it is best simply to discuss the observed value of $I_{1}$, formed by the whole atmosphere along the line of sight.

B. PAGEL: I agree with Thomas.

J. HoutGAST:I admit that the effect of the chromosphere has to be taken into account more fundamentally: but subtraction of the chromospheric emission due to the chromosphere outside the limb has to be made in any case; as to the part of the chromosphere projected on the solar disk, my treatment must be seen as a first order correction. Support for this is given in the following remark by Jefferies.

J. T. JEFFERIES: In this connection I would like to draw attention again to the extremely interesting behaviour of some of the rare earth lines which persist a very long way in emission into the disk. This was pointed out by Menzel in 1934 and in spite of its apparent significance has remained unexplained and almost ignored.

J. C. PECKER: I am surprised to hear that lines should be in emission within $1000 \mathrm{~km}$ of the limb: but optical depth of the photosphere along the line of sight must be extremely great much closer to the limb than $1000 \mathrm{~km}$; actually it is equal to 1 at the limb itself.

J. Houtgast: The emission, according to computations based on a model with absorption, sets in only about $100 \mathrm{~km}$ within the limb. But the observations with a slitless spectrograph show the emission lines so long as the Moon's edge is within $1000 \mathrm{~km}$ from the limb. This is demonstrated in Fig. 8 .

D. V. Thomas: Has Dr. Houtgast made any allowance for the effect of "seeing" at the time of the eclipse?

J. HoutgaSt: "Seeing" has no effect on equivalent widths and only a small at random influence on the absolute intensities of spectral lines.

J. C. PECKER: I have noted on Dr. Houtgast's slides that the emission did not start at the same distance from the limb for all ion lines: is this effect objective, and if so, what is the reason: different excitations, or what else?

J. HoutGast : Dependence of the point of reversal from absorption to emission on excitation-potential or other parameters will possibly be determined if the whole of our observational data can be considered. The effect in itself stands without doubt.

$C$. DE JAGER: What one should like to have is the variation of the central intensity $I_{0}$ of the lines across the disk and beyond it. In order to obtain this function the observations should be subjected to two operations: (a) "differentiation" of the crescent intensities in order to obtain the surface brightness; (b) reduce the line profiles for the influence of the apparatus profile.

Do the observations allow these operations?

J. Houtgast: Differentiation will be possible, but line profiles of those weak lines cannot be accurately obtained from our observations.

G. ELSTE: The equation for the line intensity in the case of weak lines, i.e. for a small value of $\tau_{r}$ can be split up into two parts, one for the chromosphere and one for the photosphere.

$$
I=\int_{0}^{\tau_{\nu}} S\left(\tau^{\prime},\right) \exp \left(-\tau^{\prime}, \mu\right) \frac{\mathrm{d} \tau^{\prime}{ }_{\nu}}{\mu}+\int_{\tau_{\nu}}^{-\infty} S\left(\tau^{\prime}{ }_{\nu}\right) \exp \left(-\tau^{\prime}{ }_{\nu} / \mu\right)-{ }_{\mu}
$$

I don't sce any reason why that should not be possible.

D. V. Thomis: The source function must be known. 